\title{
4 STEPS FOR FIGHTING COVID-RELATED ANXIETY: AN APPLICATION OF VIRTUAL REALITY IN A SMALL COMPANY
}

\author{
Diletta Mora ${ }^{1}$, Alessandra Falco ${ }^{2}$, Annamaria Di Sipio ${ }^{2}$, \& Alessandro De Carlo ${ }^{1}$ \\ ${ }^{1}$ Lumsa University of Rome (Italy) \\ ${ }^{2}$ University of Padua (Italy)
}

\begin{abstract}
The need to effectively fight against work-related stress and anxiety, especially due to the COVID-19 outbreak, is crucial. Employees have been faced with two options: adapting to the online environment or risking contagion in public locations--both stressful conditions. Therefore, recovery actions were requested by organizations. To understand recovery processes, refer to the Effort-Recovery Model and the Theory of Conservation of Resources. According to the literature, one should not be excessively exposed to work demands, but, conversely, acquire new resources, including personal ones, to recover those that have been lost. Recovery processes can be initiated through what we call recovery experiences. In recent years, literature and practice have been enriched with contributions about the use of virtual reality (VR) as a tool for combating anxiety disorders, reducing stress, and developing soft skills.

VR proposes a technology that allows people to be immersed in a virtual environment and to interact with different stimuli: it can be used in combination with psychology techniques to improve health and well-being.

A four-step protocol, based on VR, was proposed to a small private company to improve health and performance by learning specific recovery techniques; the protocol aimed to reduce the levels of work-related stress and anxiety, in addition to enhancing personal resources such as resilience, stress management, and self-efficacy.

The participants were the employees and managers of the company $(\mathrm{N}=14)$ who were administered a four-week training protocol comprising four one-hour VR-based sessions. Two sessions (the first and the third) focused on body consciousness, while the other two were psychological techniques ("Virtual Three Good Things" and "Best Possible Self").

The obtained data showed a decrease in anxiety and stress and an improvement in personal resources. Data also showed greater effectiveness of the VR-based protocol compared to similar interventions conducted without VR. Qualitative observation is relevant as it shows a great emotional impact of the VR-based protocol, as well as a high perception of efficacy.

The limitations of the study are primarily related to the number of participants: further restrictions due to a regional worsening of the pandemic made an intermission necessary. Agreements are already in place with the parent company to encorauge more applications.

The objectives and the protocol can be a useful contribution to support employees in managing stress. VR technology can greatly help psychologists to be effective in organizations.
\end{abstract}

Keywords: Virtual reality, recovery, stress management, personal resources, organizational well-being.

\section{Introduction}

Since December 2019, the world has been facing a new contagious disease, COVID-19. It has created an unprecedented global crisis, requiring drastic changes to living conditions, social life, personal freedom, and economic activity.

Recent studies showed that the impact of the pandemic has been more severe for employees most exposed to the disease and for employees who work in close quarters with other colleagues, while the effects have been significantly less severe for employees who can work remotely (Lee, 2020).

An increase in the level of anxiety associated with COVID-19 as well as general somatic symptoms, in particular gastrointestinal conditions and fatigue, were reported (Beland et al., 2020).

Work and organizational psychology has a long history in private and public companies. Its ultimate goal is to improve the well-being, health, and performance of employees, both individually and as a group. Considering the data provided by the World Health Organization - WHO, which highlight anxiety and stress as an important risk factor for the well-being of employees and the company in general, 
it is essential that preventive, training, and intervention measures are adopted at the work process management level (Dal Corso et al., 2020).

Several studies have investigated the negative effects of work-related stress. The results showed that it is a strong predictor of illness, accidents at work, absenteeism, poor performance, high turnover, interpersonal conflicts and, above all, mental disorders, particularly mood disorders. Typical work-related stressors include role ambiguity, workload, and a low level of autonomy. Work-related stress is also reported to be associated with possible biomarkers of stress (Falco et al., 2018).

\section{Virtual reality}

The new technologies play a growing and central role in our society and are also promising ways of working and caring for employees in the psychological field. A special category is virtual reality (VR).

It consists of a human-computer interface that allows the person to interact dynamically with the virtual environment, making live immersive and interactive experiences in a three-dimensional context.

Between 1992 and 1999 a series of studies was conducted on the use of VR for the treatment of psychological disorders related to phobias and, more generally, to anxiety (Botella et al., 2004). Other studies investigated chemotherapy-derived stress (Schneider et al., 2011), pain reduction (Espinoza et al., 2012), post-traumatic stress disorder (Beidel et al., 2019).

Likewise, in the organizational context, ample scientific evidence demonstrated that new technologies play an important role in achieving individual and collective well-being.

Several studies show that VR can be a valid tool for developing team working and entrepreneurial skills useful within a work team (Kiss et al., 2016) as well as an aid for relaxation techniques (Anderson et al., 2017). VR can improve various activities related to work and organizational psychology. Its effectiveness can be observed in primary (i.e. organizational), secondary (training/educational) and tertiary (individual and clinical support) interventions. The literature highlights that new technologies can be effective tools to reduce anxiety and work-related stress also through relaxation techniques (Anderson et al., 2017). These techniques can influence positive affectivity leading to an increase in positive emotions and a reduction in negative ones (Banõs et al., 2013).

The main theories used to create the protocols are the Effort-Recovery Model (E-R) (Meijman $\&$ Mulder, 1998) and the Conservation of Resources Theory (COR) (Hobfoll, 1998). The former focuses mainly on the consequences of workload: high work demands and excessive workload lead to fatigue, a high level of irritability, and a lack of motivation to put forth new efforts. The concepts of energy and fatigue are predominant in the model.

The concept of recovery mainly refers to the reduction or elimination of the symptoms of fatigue and the restoration of optimal energy levels. Restoring ideal energy levels allows to accept new work demands while, if the energy level is not restored, irreversible consequences related to the psychophysical health of the person may follow.

The basic principle of COR, on the other hand, is based on the assumption that people struggle to obtain, preserve, and protect their resources, which can be external, such as economic goals or rewards, or internal, such as their own energies or personal characteristics.

On these solid scientific foundations, an innovative project was designed involving psychologists and psychotherapists in the creation of a path of VR-based psychological support. The goal of the resulting protocol is to provide employees and managers with techniques to reduce the levels of work discomfort, anxiety, and work-related stress, as well as to enhance psychological capital. The protocol was applied right after validation to doctors and nurses working at the COVID-19 Hospital in Schiavonia (near Padua) during the first lockdown in response to the pandemic (April 2020). The results exceeded expectations: the VR-based protocol proved to be efficient in terms of costs and time with just four effective and cutting-edge sessions.

\section{The study}

Four VR-based sessions were created. The first focused on the practice of diaphragmatic breathing, a type of deep breathing in which the diaphragm is involved. In the first part of the session, a semi-structured interview is conducted and the participant's working environment is investigated. Then, the different aspects related to stress management are explored; finally, diaphragmatic breathing is taught.

The second session took into consideration past successes and achievements. After a brief review of the diaphragmatic breathing technique, the training is devoted to the "Virtual Three Good Things" exercise: the participant is asked to describe a past success while being (virtually) present where it happened. The power of VR helps to relive a moment of success. Remembering positive elements of one's experience helps to tune in to the positive dimension of life. This exercise is useful because it can change one's approach to events: an event is stressful if it is perceived as such. 
The third session focused again on the physical aspects of stress and taught a technique called Body Focus, based on mindfulness. This technique allows to regulate emotions and reduce stress; VR helps the participant focus on the present moment, by helping to clear the mind of intrusive thoughts.

Finally, the fourth session focused on future positive thinking. Through the technique called the "Best Possible Self" the participant will be guided along three different virtual environments to stimulate different reflections on the best possible self. The participant will reflect on the goals and the skills needed to achieve them. This will help to put into practice concrete actions to build new skills to achieve goals and results (De Carlo et al., 2020).

The aim of the four training sessions is to achieve the following objectives:

- increasing and improving health and performance in the workplace by teaching and learning specific techniques and strategies that can be used independently by participants even outside the workplace;

- reducing the levels of work-related stress, anxiety, and work discomfort thanks to the use of specific intervention protocols;

- monitoring pre- and post-intervention anxiety levels through VR;

- enhancing individual resources such as resilience, stress management, and self-efficacy, or the individual's awareness of possessing the skills needed to achieve specific goals and work results.

\section{Method}

A total of 14 participants took part in the present study, all employees at a small private company. Three intervention groups were identified: five participants were exposed to the anxiety and stress management protocol; five participants received training in the same techniques in non -VR- relevant environments, and the last four participants learned the same techniques without the use of VR. After giving informed consent, participants were administered three self-report questionnaires (STAI-Y1, STAI-Y2, users' satisfaction questionnaire, and virtual environment perception questionnaire) to investigate anxiety levels and satisfaction in the workplace. Subsequently, the VR-based training began. It consisted of four different sessions, each on a separate day aimed to teach cognitive and behavioral techniques useful to reduce and manage anxiety and stress.

The sessions lasted 50-60 minutes. At the end of each, an explanation of the exercise was given together with instructions for practicing it at home. A form was also provided for monitoring the exercise in order to be able to discuss it in the next session, also addressing the emergence of any problems.

The following overall self-report psychological questionnaires were employed: STAI-Y1 (state anxiety) before and after each session and STAI-Y2 (trait anxiety) before the first session and after the last one. These questionnaires were administered to assess the level of anxiety participants experienced. Both questionnaires comprise 20 items investigating either the level of anxiety experienced by the respondents at the moment of answering (STAY-Y1) or their general level of anxiety (STAY-Y2). A users'satisfaction questionnaire was administered ad-hoc to investigate the satisfaction of participants after watching the VR video. Finally, a virtual environment perception questionnaire was administered to investigate the participant's perception of "presence", defined as the sensation of being in a place — in the case of an immersive VR environment the sensation of being in the virtual world - "you are there" (De Carlo et al., 2020).

\section{Data analyses, interpretation, and results}

Our finding shows an overall reduction in the level of anxiety measured after the exposure to VR compared to the levels assessed before the experience. In all groups and in all four sessions there was a reduction in scores related to state anxiety. This improvement was greater after exposure to VR than in the control condition (without VR).

Table 1. Pre and post $1^{\text {st }}$ session results - state anxiety.

\begin{tabular}{|c|c|c|}
\hline Group & Pre & Post \\
\hline VR & 1.58 & 1.18 \\
\hline VR neutral & 1.93 & 1.26 \\
\hline No VR & 1.75 & 1.53 \\
\hline
\end{tabular}

Table 3. Pre and post $3^{\text {rd }}$ session results - state anxiety

\begin{tabular}{|c|c|c|}
\hline Group & Pre & Post \\
\hline VR & 1.86 & 1.41 \\
\hline VR neutral & 1.83 & 1.31 \\
\hline No VR & 2.11 & 1.78 \\
\hline
\end{tabular}

Table 2. Pre and post $2^{\text {nd }}$ session results - state anxiety.

\begin{tabular}{|c|c|c|}
\hline Group & Pre & Post \\
\hline VR & 1.54 & 1.27 \\
\hline VR neutral & 2.07 & 1.68 \\
\hline No VR & 1.87 & 1.65 \\
\hline
\end{tabular}

Table 4. Pre and post $4^{\text {th }}$ session results - state anxiety.

\begin{tabular}{|c|c|c|}
\hline Group & Pre & Post \\
\hline VR & 1.45 & 1.22 \\
\hline VR neutral & 1.78 & 1.61 \\
\hline No VR & 2.40 & 1.60 \\
\hline
\end{tabular}


The results referring to the reduction of trait anxiety are also relevant. Tables 5 and 6 show a variation: our intervention led to a variation of the psychological strain and of trait anxiety.

Table 5. Pre- and post-training results - trait anxiety.

\begin{tabular}{|l|l|l|}
\hline Group & $\begin{array}{l}\text { Pre-training } \\
\text { anxiety }\end{array}$ & $\begin{array}{l}\text { Post-training } \\
\text { anxiety }\end{array}$ \\
\hline VR & 2.48 & 2.31 \\
\hline
\end{tabular}

Table 6. Pre- and post-training results - psychological strain

\begin{tabular}{|l|l|l|}
\hline Group & $\begin{array}{l}\text { Pre-training } \\
\text { strain }\end{array}$ & $\begin{array}{l}\text { Post-training } \\
\text { strain }\end{array}$ \\
\hline VR & 1.88 & 1.29 \\
\hline
\end{tabular}

Participants' satisfaction scores were high. In VR conditions the level of satisfaction increased in the course of the intervention, with the average score of 9.20 recorded half-way through reaching 9.60 out of 10-point scale at the end of the intervention. Average satisfaction score without VR was 7.70.

As mentioned above, the sense of presence was investigated and the average value reported was 4.50 on a 5-point scale. This data indicates that the participants perceived the virtual environment as real, believing they were part of it and having the sensation of touching the objects and perceiving the stimuli present in the virtual world.

The sense of presence experienced allows the participant to quickly and effectively learn the techniques taught during the four training sessions.

The qualitative data are also interesting. In all four sessions the compliance level is high and constant for the group treated with VR. $100 \%$ of the participants implemented one or more techniques learned during the training sessions. Only $13 \%$ of the participants in the non-VR group practiced these techniques.

The participants' comments about their experience were significant. They reported benefits related to the treatment, such as a greater sense of relaxation, quietness, well-being, as well as a greater ability to manage tough situations:

"I feel relieved, I felt benefits, I feel relaxed"

"I feel relaxed, almost asleep, very calm. VR is beautiful"

"I feel good, more relaxed, and free-minded"

"It's really good, everyone should do it"

"I really like the feeling: it gets all of the bad thoughts out of my head, they leave together with the blue smoke coming out...nice experience!"

"I felt calmer, I felt like I was doing something nice for my well-being"

As anticipated, all the participants exposed to VR practiced the techniques learned during the training in their daily life and to counteract stressful events. Below are some of their comments:

"I did the breathing at least 4/5 times when I was anxious and it helped!"

"I practiced breathing daily and autonomously in times of anxiety"

"I practiced breathing daily, even on Sunday in the pool"

"I practiced breathing sometimes in the morning, other times in the evening, sometimes even outside the daily exercises"

"It was nice, I feel relaxed. I thought it would make me tense and instead it was nice"

"I feel good, calmer, and free-minded"

"At the beginning of the exercise (body focus) I felt a stone on one foot, a sense of heaviness. In the end, however, I felt like I was in a stream: clear, light and free"

"At the beginning I thought I was relaxed, but at the end of the exercise I discovered a sense of relaxation that I had never felt before"

"I used to feel heavy, but now I feel like I am inside a sphere of water, I feel a sensation of warmth and protection"

As can be seen from the participants'comments, satisfaction was very high and what emerged most is the effectiveness of diaphragmatic breathing, that people reported using daily when feeling anxious. They claimed benefiting from it and feeling lighter and freer. VR was perceived as beautiful and captivating. The training was also perceived by the participants as a good deed toward themselves. One of the advantages of VR is the immersion, in fact the participants were able to live very intense experiences, as if they were inside the virtual environment, which greatly enhanced the beneficial effect of the training. 


\section{Conclusions}

Thanks to VR tools it has been possible to support employees in managing work-related stress, but also the lockdown period which in recent months has drastically changed the way of working. The positive responses received after the four training sessions highlight how important it is to focus on the employees' well-being, which leads to an improvement in both the climate within the organization and performance.

Training led to the enhancement of employees' personal resources, such as resilience, optimism, self-efficacy, which VR does more effectively and faster than traditional methods.

The consistent results obtained proved that the set objectives can be reached and that this VR-based training can help employees to assume new points of view to manage anxiety and stress as well as enhancing individual resources and creating a positive atmosphere in the work environment.

Given the limited number of employees who participated in the study, mainly because of the COVID-19-related restrictions, this work is still an ongoing experience. Nevertheless, the results already obtained suggest that this area of intervention and this protocol have a potential that should be further investigated and replicated in broader contexts.

\section{References}

Anderson, A. P., Mayer, M. D., Fellows, A. M., Cowan, D. R., Hegel, M. T., \& Buckey, J. C. (2017). Relaxation with immersive natural scenes presented using virtual reality. Aerospace medicine and human performance, 88(6), 520-526.

Baños, R. M., Espinoza, M., García-Palacios, A., Cervera, J. M., Esquerdo, G., Barrajón, E., \& Botella, C. (2013). A positive psychological intervention using virtual reality for patients with advanced cancer in a hospital setting: A pilot study to assess feasibility. Supportive Care in Cancer, 21(1), 263-270.

Beidel, D. C., Frueh, B. C., Neer, S. M., Bowers, C. A., Trachik, B., Uhde, T. W., \& Grubaugh, A. (2019). Trauma management therapy with virtual-reality augmented exposure therapy for combat-related PTSD: A randomized controlled trial. Journal of anxiety disorders, 61, 64-74.

Beland, L. P., Brodeur, A., Mikola, D., \& Wright, T. (2020). The short-term economic consequences of Covid-19: Occupation tasks and mental health in Canada. IZA Discussion Papers, Article 13254.

Botella, C., Hofmann, S. G., \& Moscovitch, D. A. (2004). A self-applied, Internet-based intervention for fear of public speaking. Journal of clinical psychology, 60(8), 821-830.

Dal Corso, L., De Carlo, A., Carluccio, F., Colledani, D., \& Falco, A. (2020). Employee burnout and positive dimensions of well-being: A latent workplace spirituality profile analysis. PloS One, 15(11), article e0242267. doi:https://doi.org/10.1371/journal.pone.0242267

De Carlo, A., Carluccio, F., Rapisarda, S., Mora, D., \& Ometto, I. (2020). Three uses of virtual reality in work and organizational psychology interventions. a dialogue between virtual reality and organizational well-being: relaxation techniques, personal resources, and anxiety/depression treatments. TPM - Testing, Psychometrics, Methodology in Applied Psychology, 27(1), 129-143. doi: 10,4473/TPM27.1.8

De Carlo, A., Rapisarda, S., Mora, D., \& Benevene, P. (2020, April). Your best virtual self-improvement. The use of virtual reality in stress management training [Paper presentation]. International Psychological Applications Conference and Trends - InPACT 2020.

Espinoza, M., Baños, R. M., García-Palacios, A., Cervera, J. M., Esquerdo, G., Barrajón, E., \& Botella, C. (2012). Promotion of emotional wellbeing in oncology inpatients using VR. Annual Review of Cybertherapy and Telemedicine, 53-57.

Falco, A., Dal Corso, L., Girardi, D., De Carlo, A., \& Comar, M. (2018). The moderating role of job resources in the relationship between job demands and Interleukin-6 in an Italian healthcare organization. Research in Nursing \& Health, 41, 39-48.doi:10.1002/nur.21844

Hobfoll, S. E. (1998). Stress, culture, and community: The psychology and physiology of stress. New York: Plenum Press.

Kiss, L., Hámornik, B. P., Köles, M., Baranyi, P., Galambos, P., \& Persa, G. (2015, October). Training of business skills in virtual reality. In 2015 6th IEEE International Conference on Cognitive Infocommunications (CogInfoCom) (pp. 215-216). IEEE.

Lee, S. A. (2020). Coronavirus Anxiety Scale: A brief mental health screener for COVID-19 related anxiety. Death studies, 44(7), 393-401.

Meijman, T. F., \& Mulder, G. (1998). Psychological aspects of workload. In P. J. D. Drenth, \& H. Thierry (a cura di), Handbook of work and organizational psychology (Vol. 2: Work psychology, pp. 5-33). Hove, England: Psychology Press.

Schneider, S. M., Kisby, C. K., \& Flint, E. P. (2011). Effect of virtual reality on time perception in patients receiving chemotherapy. $19(4), 555-564$. 